\title{
Affective picture perception: gender differences in visual cortex?
}

\author{
Dean Sabatinelli, ${ }^{\text {CA }}$ Tobias Flaisch, Margaret M. Bradley, Jeffrey R. Fitzsimmons and Peter J. Lang
}

NIMH Center for the Study of Emotion and Attention, P.O. Box 100165 HSC, University of Florida, Gainesville, FL, USA 32610, USA

${ }^{\mathrm{CA} C}$ orresponding author: sabat@ufl.edu

Received 2I November 2003; accepted 4 March 2004

\begin{abstract}
Activity in extrastriate visual cortex is greater when people view emotional relative to neutral pictures. Prior brain imaging and psychophysiological work has further suggested a bias for men to react more strongly to pleasant pictures, and for women to react more strongly to unpleasant pictures. Here we investigated visual cortical activity using $\mathrm{fMRI}$ in 28 men and women during picture viewing. Men and women showed reliably greater visual cortical reactivity during both pleasant and unpleasant pictures, relative to
\end{abstract}

neutral, consistent with the view that the motivational relevance of visual stimuli directs attention and enhances elaborative perceptual processing. However, men did show greater extrastriate activity than women specifically during erotic picture perception, possibly reflecting a gender-specific visual mechanism for sexual selection. NeuroReport 15:I109-III2 (c) 2004 Lippincott Williams \& Wilkins.

Key words: Attention; Emotion; fMRI; Gender differences; Pictures; Visual cortex

\section{INTRODUCTION}

Visual cortical activity, as measured by blood oxygen level dependent (BOLD) signal or cortical event related potentials (ERP), varies systematically with picture content: Viewing highly arousing pictures (erotica, physical threat) evokes greater activation than viewing moderately arousing pictures (families, babies), which in turn result in more activity than neutral picture perception (expressionless faces, household objects) [1-4]. These differences are found regardless of whether pictures are presented in full color or grayscale [1], and when picture sets are balanced for physical characteristics such as brightness, contrast, and spatial frequency $[4,5]$. Thus the level of visual cortical activation in part reflects the motivational relevance of the visual scene, presumably via its dense interconnections with subcortical structures involved in defensive and appetitive responding [6-8]. This natural selective attention may be an evolutionary adaptation promoting enhanced processing of potentially life threatening or life sustaining input $[9,10]$.

While these general brain activation patterns are shared by both men and women, studies of verbal report and physiological activity have found intensity differences in the reactions of men and women to emotional stimuli [11-14]. Men rate erotica as more pleasant and arousing than do women, and respond with greater sympathetic (skin conductance) activity when viewing erotic pictures, whereas women rate aversive pictures as more unpleasant and arousing than do men, and respond with greater facial muscle tension and fear bradycardia [11,14]. Cortical measures of visual processing, including ERP [15] and MEG [16] also suggest that men are more reactive to pictures of the opposite sex than are women. These differences are thought to reflect a combination of biological and cultural influences, including visually oriented sexual selection criteria in men, and heightened defensive vigilance in women $[11,13,14,17]$.

Functional imaging studies have also reported gender differences in affective picture perception. In an fMRI study of blocked pleasant, neutral, and unpleasant picture perception, women showed greater activation in the right extrastriate visual cortex when viewing unpleasant compared to pleasant pictures, whereas men showed the opposite trend [3]. A more recent fMRI study that also assessed pleasant, neutral, and unpleasant pictures reported that, compared to women, men showed greater fusiform gyrus (and amygdala) activation for the pleasant pictures [18].

In the research reported here, visual cortical BOLD reactivity was measured in a substantial sample of men and women, viewing a range of affective and neutral picture categories (including erotic couples, families, household objects, neutral faces, angry faces, physical threat, and mutilated bodies). The arousing picture contents were chosen because they have produced reliable gender-specific responses in previous psychophysiological analyses [14]. On this basis, it was hypothesized that men would show greater functional activity in visual cortex than women when viewing pictures of erotica, while women would show greater visual cortical activation than men when viewing arousing, unpleasant pictures (threatening people or animals and mutilated bodies).

\section{MATERIALS AND METHODS}

Participants: Twenty-eight introductory psychology students (14 men, mean ( \pm s.d.) age 18.6 \pm years; 14 women, mean age $18.3 \pm 1.4$ years), screened for claustrophobia and 
physical restrictions, chose to participate after reading through a consent form approved by the University of Florida Institutional Review Board. Data from the 14 male subjects were a subset of those previously published in a study focusing on physical and semantic picture effects [1].

Materials and design: Stimuli consisted of 56 pictures, selected from the International Affective Picture System [19] and from the Japanese and Caucasian Facial Expressions of Emotion set [20], divided into seven categories, including eight pictures each of erotic couples, families, household objects, neutral faces, angry faces, physical threat, and human injury/mutilation. The International Affective Picture System is available on CD-ROM and as photographic slides. The IAPS and technical manuals can be obtained on request from the authors at the NIMH Center for the Study of Emotion and Attention, Box 100165 HSC, University of Florida, Gainesville, FL 32610-0165, USA.

Each of seven picture category blocks consisted of eight cycles of a $12 \mathrm{~s}$ presentation period, with pictures flickering at $3 \mathrm{~Hz}$, and a $12 \mathrm{~s}$, dark screen inter-picture interval. Order of picture content blocks was counterbalanced across participants.

MR data acquisition: Images were acquired using a custom-built quadrature head coil designed to increase the signal-to-noise ratio in the occipital region, including a bite bar to minimize head motion [21]. Subjects viewed the picture stimuli (subtending a $20^{\circ}$ visual angle) on a screen mounted at their feet, via a coil-mounted mirror. Scanning began with a T1-weighted sagittal volume, which was used to prescribe seven coronal functional slices, originating $10 \mathrm{~mm}$ anterior to the occipital pole. Functional images were acquired with a 4-shot spiral scan sequence [22] on a $3 \mathrm{~T} \mathrm{GE}$ Signa scanner $\left(10 \mathrm{~mm}^{3}\right.$ voxels, TE 35 , TR $\left.2880 \mathrm{~ms}\right)$, yielding 68 images for each of the seven picture blocks.

Procedure: Subjects were asked to lie still and fixate centrally on a laser point during the seven picture blocks, lasting $\sim 22$ mins. Two-way communication was open throughout the session.

Data reduction and analyses: Functional slices were reoriented, motion-corrected, resampled to isomorphic $2 \mathrm{~mm}$ voxels, co-registered with the structural volume, and standardized to the Montreal Neurological Institute template (SPM99; Wellcome Department of Cognitive Neurology, London, UK). A $12 \mathrm{~mm}$ FWHM smoothing kernel was applied to the functional data. Effects were evaluated using the general linear model, with a voxel-wise uncorrected probability threshold of $\alpha=0.001$, and a minimum cluster size of 50 voxels. Co-variates included a hemodynamic response-convolved boxcar function and its first derivative. After individual model estimation, contrasts comparing conditions of interest were defined and calculated, and entered into a second-level random effects group analysis.

Region of interest analyses: A region of interest (ROI) was defined including a contiguous cluster of 16259 voxels of significant activation during all picture presentations compared with the dark screen inter-picture interval (Fig. 1a). This ROI was divided at the midline into right (8008 voxels) and left (8251 voxels) portions. Signal change measures were determined by averaging signal intensity values within left and right ROIs for each time point, and computing a change score for each picture within a block. These scores were then averaged across the eight pictures within each of the seven categories by subject, and standardized (t-score) within hemisphere according to each subject's mean and standard deviation. These values were entered into a $2 \times 2 \times 7$ ANOVA including factors for gender, hemisphere, and picture category.

\section{RESULTS}

Replicating prior studies, greater visual cortical activation was elicited during presentation of emotional (erotica, families, angry faces, threat, and mutilation), compared to neutral pictures (household objects and neutral faces; 1754 voxels; see Fig. 1b). Compared with neutral pictures, viewing emotionally arousing pictures led to greater activation in middle occipital, inferior occipital, and fusiform gyri.

Men and women showed a similar general pattern of BOLD activation over picture content categories although, as can be seen in Fig. 2, the difference volumes tended to be consistent with the gender hypotheses. A region of interest analysis was used to investigate signal change in these areas and test the experimental hypotheses.

ROI analysis: As expected, signal change in the visual cortical ROI was reliably modulated by picture category $(F(6,156)=12.60, p<0.001)$, with arousing picture categories eliciting greater BOLD signal change (Fig. 3). Men and women were again generally similar in response to the picture stimuli (gender $\times$ category $\mathrm{F}(6,156)=1.24$, ns); and signal change varied significantly by picture category for both women $(\mathrm{F}(6,78)=7.99, p<0.001)$ and men $(\mathrm{F}(6,78)=5.94, p<0.001)$, with no interaction. Picture content modulated signal change consistently in the two hemispheres (category $\times$ hemisphere $\mathrm{F}(6,156)=1.36$, ns), though greater overall signal change was elicited in the right compared to the left (hemisphere $\mathrm{F}(6,156)=10.39, p<0.01$ ).

Planned tests of specific picture contents found greater activation when participants viewed erotic, compared with family pictures $(\mathrm{F}(1,26)=7.62, p=0.01)$. This effect differed for men and women $(\mathrm{F}(1,26)=5.93, p<0.05)$ : Men showed stronger signal change when viewing erotic compared with family pictures $(F(1,13)=12.23, p<0.01)$, whereas BOLD signal during these contents did not differ for women $(\mathrm{F}(1,13)<1, \mathrm{~ns})$. Men also showed greater relative ROI signal change than women specifically during erotic pictures $(t=2.44, p<0.05)$, but did not differ from women during family pictures $(t=1.25$, ns; Fig. 3). Signal change during family pictures was greater than signal change during pictures of household objects $(\mathrm{F}(1,26)=4.54, p<0.05)$, neutral faces $(F(1,26)=11.97, p<0.01)$, and angry faces $(F(1,26)=10.01$, $p<0.01)$. None of the latter three contents differed from each other, nor were there any differences for men and women.

Signal change during threat and mutilation pictures were large and statistically equivalent, and both of these unpleasant, arousing contents yielded a greater signal increase than face stimuli or pictures of household objects. Although mean signal change during threat and mutilation pictures were larger for women than for men, direct statistical tests were not significant.

In a final assessment of the gender hypothesis, differences in ROI activation during erotic pictures and combined 


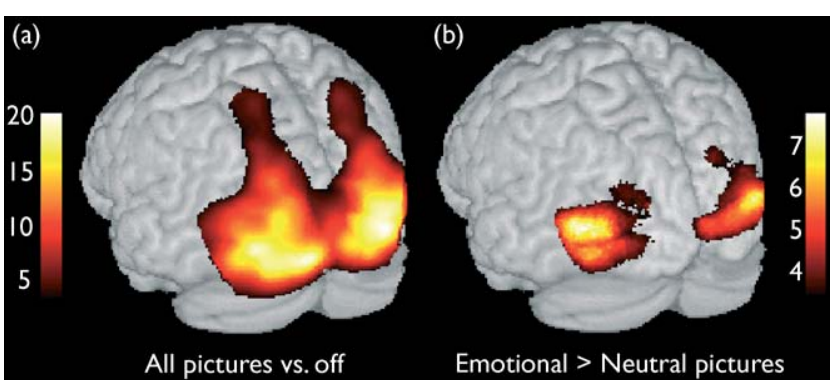

Fig. I. (a) Posterior view of functional activity elicited by picture viewing, relative to the dark-screen inter-picture interval, at an alpha level of 0.00 l, uncorrected, in striate, extrastriate, medial parietal, and inferior temporal visual cortex. (b) Posterior view of lateral occipital and inferior temporal activity during emotionally arousing (erotica, families, angry faces, threat, and mutilation) relative to neutral (household objects, neutral faces) picture perception at an alpha level of 0.00 , uncorrected.

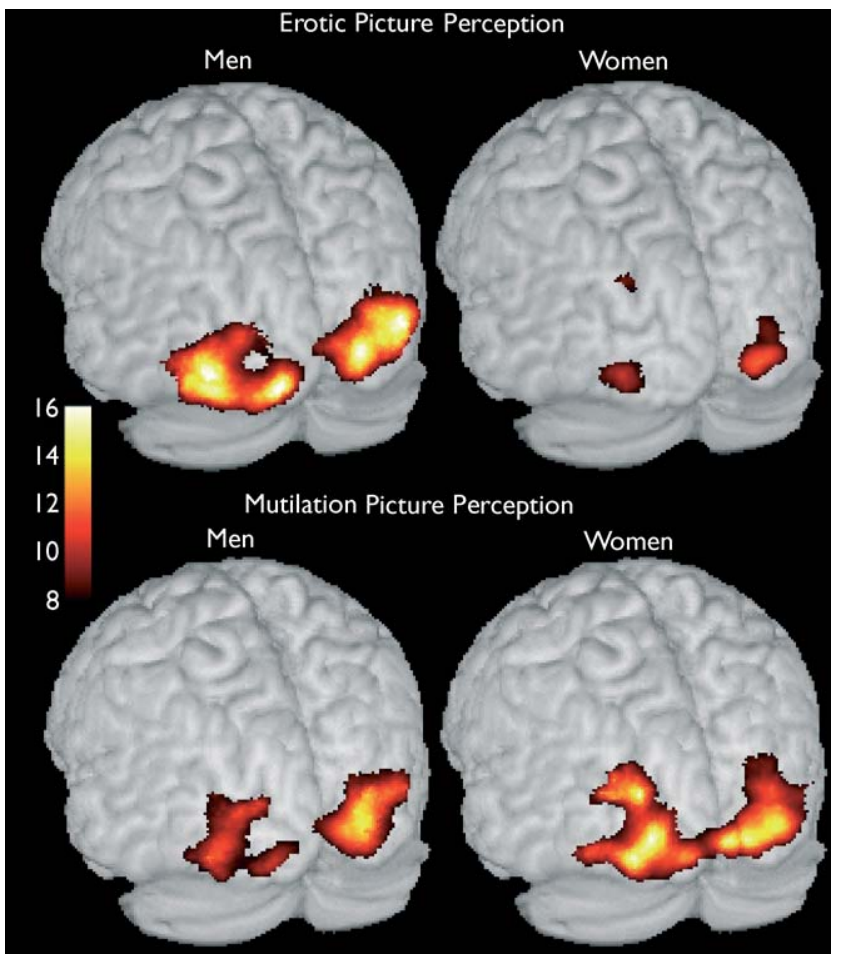

Fig. 2. Top: Functional activity during erotic picture viewing, relative to the dark-screen inter-picture interval, for men (left) and women (right), at an alpha level of 0.00000 l, uncorrected. Bottom: Functional activity during mutilation picture viewing, relative to the dark-screen inter-picture interval, for men (left) and women (right), at an alpha level of 0.00000 , uncorrected.

aversive pictures (threat and mutilation) were analyzed for men and women (Fig. 4). The category $\times$ gender statistic was significant $(\mathrm{F}(1,26)=5.79, p<0.05)$. In subsequent tests, men showed a significantly greater signal change during erotic, compared to aversive pictures $(\mathrm{F}(1,13)=4.96, p<0.05)$; signal change means for women again showed an opposite pattern, but again the difference was not significant.

\section{DISCUSSION}

Men and women showed highly similar patterns of visual cortex activation during affective picture viewing; arousing pictures led to greater visual system BOLD response than did neutral pictures, specifically in secondary sensory processing areas, including middle occipital, inferior occipital, and fusiform gyri. These effects are consistent with results from psychophysiological studies of affective picture perception that show a similar general pattern of enhanced reactivity for autonomic, somatic reflex, and electrocortical measures $[2,11,14]$. As in the current study, picture images of erotica, viewer-directed threat, and violent injury evoked the strongest responses, presumably because these stimuli are related to primary reinforcement and directly engage underlying appetitive and defensive neural systems. The fact that men and women show such comparable responses may reflect a common high-priority in the processing of survival-relevant stimuli [9-11].

Despite this uniformity in overall pattern, viewing erotic pictures did result in a significantly greater signal change in men thanin women, relative to all their other contents. This is consistent with previous findings for men, of elevated affective valence and arousal ratings, and larger skin conductance responses (relative to women) when viewing erotic pictures $[11,12,14]$. The present research permits a reinterpretation of previous fMRI studies using IAPS pictures [3,18]. It was previously inferred that men are more reactive generally to pleasant stimuli than women; the new result suggests that this interpretation depended on the inclusion of erotic content in the set of pleasant pictures. Data from this experiment suggest that women and men do not generally differ on other pleasant contents. The consistent, strong response to erotica in men, as expressed through affective ratings, sympathetic response, and visual cortical BOLD signal, suggests a specific, perhaps biologically based visual processing bias for this content. It may be an evolved species survival mechanism, optimizing mate selection (for good health, youth) [17].

Prior studies have demonstrated that women show stronger heart rate and facial muscle reactivity to aversive pictures than do men [11,13]. Some previous fMRI studies also suggest a parallel gender difference in visual cortex activation to unpleasant pictures [3]. However, the present research does not replicate these data. Interestingly, significant gender differences for unpleasant pictures are also not reliably observed in studies of the ERP [23]. The primary neural circuits mediating autonomic responses and facial muscle action are certainly more anterior and significantly deeper in the brain than the cortical volumes measured here: these are deep sites that are also difficult to assess with the electroencephalogram. It is possible that the more pronounced defense reaction in women, suggested by the physiological data [15], does not include a specific enhancement in visual processing. At the least, it appears to be a weaker phenomenon than the male bias for erotic visual stimuli. Future studies employing a wider range of aversive material or a greater number of participants may reveal significant effects.

Finally, it is noted that pictures of posed angry faces were not an effective elicitor of enhanced affect related processing in the visual cortex. This result is consistent with the findings of other picture studies in which emotional reactivity was measured by evaluative reports, facial action, and autonomic responses. In effect, posed face stimuli are not strong affect elicitors, and brain changes evoked with these stimuli are 


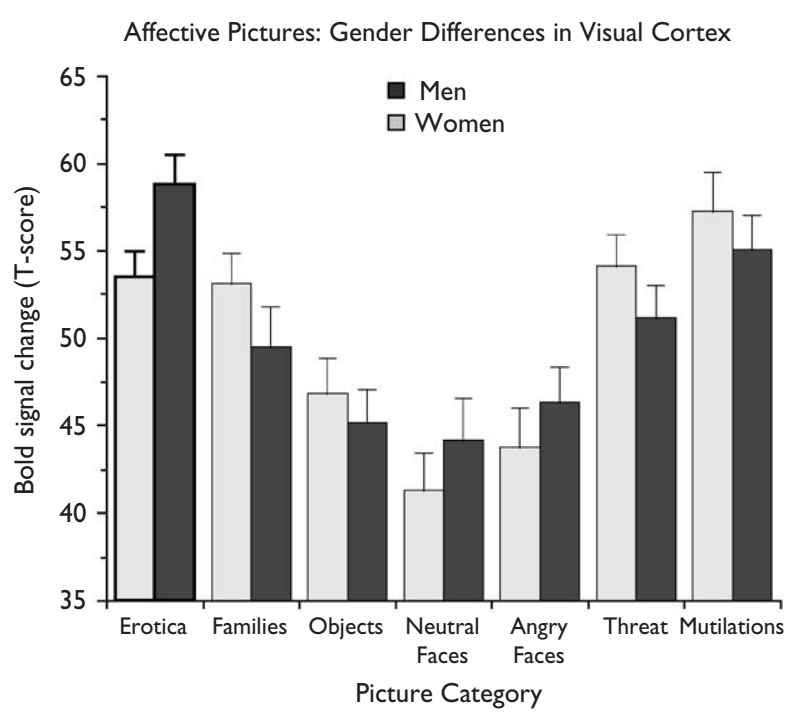

Fig. 3. Visual cortical region of interest signal change (T-score) by picture category for men (dark bars) and women (light bars).

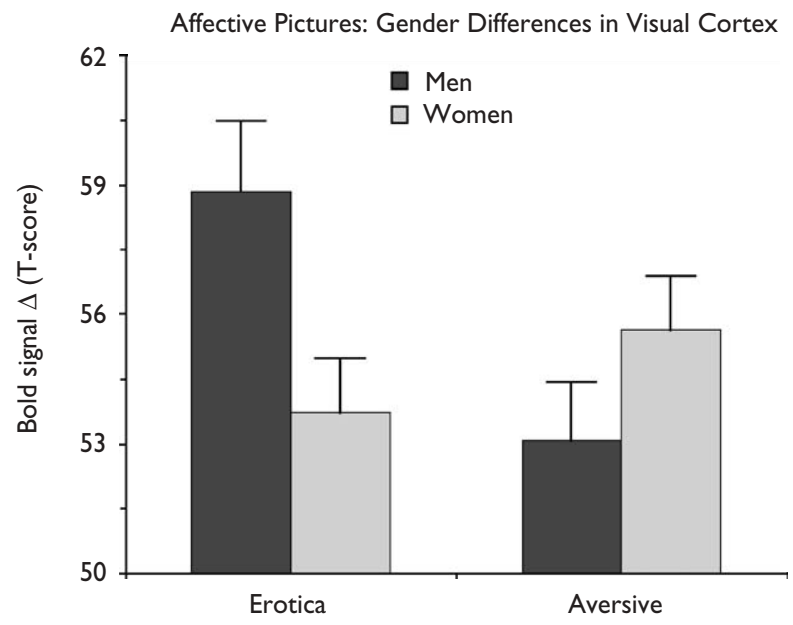

Fig. 4. Visual cortical region of interest signal change (T-score) during erotic and aversive (threat and mutilation) picture perception for men (dark bars) and women (light bars).

more likely related to circuits responsible for face discrimination than to sites relevant to emotional engagement.

\section{CONCLUSIONS}

Men and women show consistent patterns of secondary visual cortical activity while viewing a range of emotional and neutral pictures, such that motivationally relevant picture content, whether appetitive or aversive, led to greater BOLD signal. Our sample of 14 men did show greater extrastriate activity when viewing pictures of erotic couples, relative to 14 women. Future work will continue to investigate the possibility that women may show a bias toward greater visual cortical activity when viewing aversive pictures relative to men, a hypothesis that was not supported in the current dataset. Thus while the overall patterns across men and women were consistent, perhaps reflecting a common adaptation to process life-threatening and life-sustaining input efficiently, men showed a bias toward cues of sexual opportunity, possibly reflecting a gender-specific species survival mechanism.

\section{REFERENCES}

1. Bradley MM, Sabatinelli D, Lang PJ, Fitzsimmons JR, King W and Desai P. Activation of the visual cortex in motivated attention. Behav Neurosci 2003; 117:369-380.

2. Cuthbert BN, Schupp HT, Bradley MM, Birbaumer N and Lang PJ. Brain potentials in affective picture processing: Covariation with autonomic arousal and affective report. Biol Psychol 2000; 52:95-111.

3. Lang PJ, Bradley MM, Fitzsimmons JR, Cuthbert BN, Scott JD, Moulder B et al. Emotional arousal and activation of the visual cortex: an fMRI analysis. Psychophysiology 1998; 35:199-210.

4. Junghoefer M, Bradley MM, Elbert TR and Lang PJ. Fleeting images: a new look at early emotional discrimination. Psychophysiology 2001; 38:175-178.

5. Sabatinelli D, Bradley MM, Tsao JCI, Fitzsimmons JR and Lang PJ. Brain activation in emotional picture processing. Psychophysiology 2002; 39:S72.

6. Amaral DG, Price JL, Pitkanen A and Carmichael ST. Anatomical organization of the primate amygdaloid complex. In: Aggleton JP (ed.). The Amygdala: Neurobiological Aspects of Emotion, Memory, and Mental Dysfunction. New York: Wiley-Liss; 1992, pp. 1-66.

7. Shi $\mathrm{C}$ and Davis M. Visual pathways involved in fear conditioning measured with fear-potentiated startle: Behavioral and anatomic studies. J Neurosci 2001; 21:9844-9855.

8. Everitt BJ, Cardinal RN, Parkinson JA and Robbins TW. Impact of amygdala-dependent mechanisms of emotional learning. Ann NY Acad Sci 2003; 985:233-250.

9. Lang PJ, Bradley MM and Cuthbert BN. Motivated attention: affect, activation, and action. In: Lang PJ, Simons RF and Balaban MT (eds). Attention and Orienting: Sensory and Motivational Processes. Hillsdale, NJ: Erlbaum; 1997, pp. 97-135.

10. Lang PJ, Davis M and Öhman A. Fear and anxiety: animal models and cognitive psychophysiology. I Affect Disord 2000; 61:137-159.

11. Lang PJ, Greenwald MK, Bradley MM and Hamm A. Looking at pictures: affective, facial, visceral, and behavioral reactions. Psychophysiology 1993; 30:261-273.

12. Murnen SK and Stockton M. Gender and self-reported sexual arousal in response to sexual stimuli: a meta-analytic review. Sex Roles 1997; 37:135-153.

13. Kring AM and Gordon AH. Sex differences in emotion: expression, experience, and physiology. J Pers Soc Psychol 1998; 74:686-703.

14. Bradley MM, Codispoti M, Sabatinelli D and Lang PJ. Emotion and motivation II: sex differences in picture processing. Emotion 2001; 1:300-319.

15. Oliver-Rodriguez JC, Guan Z and Johnston VS. Gender differences in late positive components evoked by human faces. Psychophysiology 1999; 36:176-185.

16. Costa M, Braun C and Birbaumer N. Gender differences in response to pictures of nudes: a magnetoencephalograph study. Biol Psychol 2003; 63:129-147.

17. Buss DM. Psychological sex differences. Am Psychol 1995; 50:164-168.

18. Wrase J, Klein S, Gruesser SM, Hermann D, Flor H, Mann K et al. Gender differences in the processing of standardized emotional visual stimuli in humans: a functional magnetic resonance imaging study. Neurosci Lett 2003; 348:41-45.

19. Center for the Study of Emotion and Attention (CSEA-NIMH). The international affective picture system (IAPS; photographic slides). Gainesville, FL: The Center for Research in Psychophysiology, University of Florida; 1999.

20. Matsumoto D and Ekman P. American-Japanese cultural differences in intensity ratings of facial expressions of emotion. Motiv Emotion 1989; 13:143-157.

21. Fitzsimmons JR, Scott JD, Peterson DM, Wolverton BL, Webster CS and Lang PJ. Integrated RF coil with stabilization for fMRI in human cortex. Magn Res Med 1997; 38:15-18.

22. Noll DC, Cohen JD, Meyer CH and Schneider W. Spiral K-space MR imaging of cortical activation. J Magn Res Imag 1995; 5:49-56.

23. Schupp HT, Cuthbert BN, Bradley MM, Hillman $\mathrm{CH}, \mathrm{Hamm}$ AO and Lang PJ. Brain processes in emotional perception: motivated attention. Cogn Emotion, in press. 